\title{
Evaluation of HSV-1 and adenovirus vector-mediated infection, replication and cytotoxicity in lymphoma cell lines
}

\author{
XUDONG ZHANG ${ }^{1}, \mathrm{LU}_{\mathrm{ZHAO}}{ }^{2}, \mathrm{ZHIQIANG} \mathrm{HANG}^{3}, \mathrm{HONGQIANG} \mathrm{GUO}^{4}$ and MINGZHI ZHANG \\ Department of Oncology, The First Affiliated Hospital, Zhengzhou University; \\ ${ }^{3}$ Medical College, Zhengzhou University; ${ }^{4}$ Oncology Department, The Affiliated Cancer Hospital \\ of Zhengzhou University, Zhengzhou 450000, P.R. China
}

Received February 16, 2011; Accepted March 30, 2011

DOI: $10.3892 / o r .2011 .1306$

\begin{abstract}
Lymphoma is not a common focus for viral therapy as many researchers do not consider lymphoma as a suitable target for viral vectors. In the present study, the infection, replication and cytotoxicity of herpes simplex virus (HSV) and adenovirus vectors were screened and evaluated in different lymphoma cell lines. Three recombinant viruses, BAC-HSV-1-eGFP, Adv-eGFP and an NV1020-like oncolytic HSV-1 virus strain named BAC-HSV-1-eGFP-delIRs, inserted with green fluorescent protein (GFP) as a reporter, were applied to evaluate the efficiency of viral infection and replication and cytotoxicity to lymphoma cells. Four types of lymphoma cell lines (SNK-6, Jurkat, Raji and Hut 78) were examined. We found that the HSV-1 vector, BAC-HSV-1eGFP, was able to infect and replicate in the three lymphoma cell lines (Jurkat, Raji and Hut 78) and inhibit the growth of these cells more efficiently than adenovirus vector Adv-eGFP. The sensitivity of the four types of lymphoma cell lines to the viral vectors was different. The human cutaneous TL cell line Hut 78 was more sensitive to the viral vectors than the other cell lines. However, the human NK/T lymphoma cell line SNK-6 was not infected by any of the viral vectors and did not allow replication of the viruses. In conclusion, lymphoma may be a potential target for HSV-1 vector-mediated viral therapy.
\end{abstract}

\section{Introduction}

Lymphoma is the most common hematologic malignancy and comprises a heterogeneous group of malignancies consisting of different clinicopathological entities. Despite recent advances,

Correspondence to: Dr Mingzhi Zhang, Oncology Department, the First Affiliated Hospital, Zhengzhou University, Zhengzhou 450000, P.R. China

E-mail: zhaolu-xx@163.com

Key words: lymphoma, herpes simplex virus 1, adenovirus, viral therapy lymphoma is not yet curable, and death most commonly results from progressive refractory disease after treatment, as well as from the considerable toxicity of current therapeutic modalities $(1,2)$. The availability of molecular genetic technology and a more precise understanding of lymphoma offer potential strategies for the treatment of lymphoma. A number of promising therapies have been developed in the past few decades. For example, monoclonal antibody therapy (e.g. rituximab) directly targeting specific antigens of lymphoma cells has been the standard treatment for certain types of B-cell non-Hodgkin's lymphoma (NHL) $(3,4)$.

Another potential biological approach to the treatment of lymphoma is gene therapy. Viral therapy is a new form of gene therapy which is being investigated in clinical trials for many cancer types. Transducing exogenous genes in vitro using viral vector and vector-directed cell lysis have been the most common strategies for viral therapy in the treatment of cancer. Although viral therapy for the treatment of lymphoma appears promising according to a previous report, further research has been hampered by the lack of suitable vectors that can achieve high levels of infection efficiency and replication capability (5).

Viral vectors, such as herpes simplex virus (HSV), retrovirus, adenovirus (adv) and adeno-associated virus (aav) are required in many applications of gene therapy to tumor cells. To achieve successful treatment, viral vectors need to be delivered with high efficiency to target cells or produce tumor cell lysis with sufficient replication capability. Extensive research on viral therapy has achieved many significant results in preclinical and clinical trials. In China, two types of gene recombinant adenovirus gene drugs have been utilized in clinical application to treat solid tumors (6). In general, viral vectors for cancer treatment have been the focus of gene therapy. Human lymphomas, however, have not been a common focal point for the recent study of viral therapy, possibly because of their hematologic and heterogeneous characteristics (7). Infection of appropriate target cells is the first step in gene therapy using viral vectors. Many previous reports have demonstrated that most human lymphoid cells are relatively resistant to viral infection, and most viral therapies cannot transfer exogenous DNA into primary lymphocytes efficiently (8-12), therefore, more and more researchers presume that lymphoma is an unsuitable target for viral therapy, unless 
reliable and stable vector systems with a high infection efficiency of a large number of lymphoma cells and/or a high level of replication ability in lymphoma cells are developed.

However, lymphoma cells may still be attractive targets for viral therapy. First, specific genetic defects may exist in several NHLs that make their malignant cells attractive targets for viral assault. Second, for some hematological malignancies, the neoplastic cells circulate in the blood, thus a large number of tumor cells can be harvested and sorted for manipulation ex vivo. Third, there is evidence in the literature suggesting that some selected lymphocytic cell lines can be transduced efficiently with viral vectors (13-15). Fourth, it has been recognized that some viral vectors such as HSVs could infect malignant human hematopoietic cells with high efficiency $(12,16)$, and these studies suggest that lymphoma cells could be efficiently infected with HSV vectors. Finally, current technology permits scientists to genetically engineer viruses infecting and/or replicating in lymphoma cells with higher efficiency. For example, the Ad5/F35 fiber chimeric adenovirus effectively infecting B-lymphocytic malignancies may serve as a potential anticancer agent or a therapeutic vehicle in B-cell tumor treatment (17). In addition, attenuated measles viruses (MV) were confirmed to exhibit a strong oncolytic effect for lymphoma and myeloma in in vivo experiments $(7,18)$.

Since lymphomas consist of numerous histologic subtypes and may have different susceptibilities to various viral vectors, it is very important to ascertain the infection and replication ability of viral vectors for different types of lymphomas. It has been considered that HSV vectors can infect many tumor cell lines with high efficiency and may be a highly effective means of infecting lymphoma cells. There are three types of HSV vectors: amplicon, replication-incompetent vectors and oncolytic vectors. Various oncolytic HSV vectors have been applied in clinical trials, and the laboratory and clinical data have shown that HSV vectors are more reliable, effective and safer than traditional viral vectors (19). Moreover, there are few applications of HSV vectors in the research on lymphoma, therefore, it is necessary to screen and evaluate the infection and replication ability of HSV vectors for different lymphoma cell lines.

Here, recombinant viruses, BAC-HSV-1-eGFP (BH-1-G) and Adv-eGFP (Ad-G), inserted with green fluorescent protein (GFP) as a reporter, were applied to evaluate the efficiency of viral infection and replication. Virus BH-1-G was constructed using the bacterial artificial chromosome (BAC) and homologous recombination technology. Virus Ad-G was engineered by Gateway Technologies. Different types of human lymphoma cell lines (SNK-6, Jurkat, Raji and Hut 78) were infected with the recombinant viruses as noted above with different MOIs, and then the GFP expression was detected by both flow cytometric analysis and fluorescence microscopy. In addition, progeny viruses were harvested for titre determination to evaluate the replication ability. We also constructed an NV1020-like oncolytic HSV-1 virus strain named BAC-HSV-1-eGFP-delIRs (BH-1-G-dirs) with a deleted internal repeat region including one copy of RS1, RL1, RL2 gene, and the mutated UL56 and US1 genes to make it express one of the two original $\gamma 34.5$ genes allowing the virus to replicate more efficiently. Recently, the NV1020 oncolytic HSV-1 showed a significant potential for the treatment of many different types of tumors in preclinical studies in experimental animals as well as in human clinical trials $(20,21)$. In the present study we also examined the cytotoxicity of oncolytic virus BH-1-G-dirs to lymphoma cells after analysis of viral infection and replication ability. Finally, we found that HSV-1 vectors could infect and replicate in several types of lymphoma cells and inhibit the growth of these cells more efficiently than the adenovirus vector, and the sensitivities of lymphoma cell lines to viral vectors were significantly different.

\section{Materials and methods}

\section{Cells}

Lymphoma cell lines. Human BL cell line Raji, human TL cell line Jurkat and human cutaneous TL cell line Hut 78 were obtained from the laboratory of Zhang Mingzhi, The First Affiliated Hospital of Zhengzhou University. The human NK/T lymphoma cell line SNK-6 was provided by Professor Norio Shimizu and Yu Zhang of Chiba University.

SNK-6 cells were grown in RPMI-1640 with $10 \%$ human serum and $700 \mathrm{U} / \mathrm{ml}$ IL-2. Hut 78 cells were grown in RPMI1640 with $10 \%$ fetal bovine serum. Raji and Jurkat were grown in RPMI-1640 with $10 \%$ fetal bovine serum, $1.5 \mathrm{~g} / 1$ $\mathrm{NaHCO}_{3}, 10 \mathrm{mM}$ HEPES and $1.0 \mathrm{mM}$ sodium pyruvate.

Cell lines for virus manipulation. The human embryonic kidney 293A cell line and the African green monkey kidney Vero cell line were cultured in DMEM supplemented with $10 \%$ fetal bovine serum, $0.1 \mathrm{mM}$ non-essential amino acid and $2 \mathrm{mM}$ glutamine. All the culture media were supplemented with $100 \mathrm{U} / \mathrm{ml}$ streptomycin and penicillin $\mathrm{G}$, and all of the cell lines were cultured at $37^{\circ} \mathrm{C}$ in a humidified atmosphere with $5 \% \mathrm{CO}_{2}$.

Viral vectors. Recombinant BAC-HSV-1-GFP virus (BH-1-G), BAC-HSV-1-GFP-delIRs (BH-1-G-dirs) and recombinant Adv-GFP virus (Ad-G) were obtained from the laboratory of Han Zhiqiang, Medical College of Zhengzhou University (Zhengzhou, China). The HSV vectors (BH-1-G, BH-1-G-dirs) and Adv-GFP virus were propagated and titrated on Vero and 293A cells, respectively. The BAC-GFP gene was inserted into the genome of HSV-1 F strain to replace several non-essential genes according to the packaging ability of the virus. The CMV-GFP-IRES-E1a-polyA element was inserted into the expression vector involving the adenovirus genome with E1 and E3 region deleted. The schematic strategy of the viral vectors is shown in Figs. 1-3.

Preparation of recombinant virus. Vero and 293A cells were transfected with the HSV vectors (BH-1-G, BH-1-G-dirs) and the Ad-G viral vector, respectively, using Liposome 2000. The cells and supernatant were harvested until $\sim 80 \%$ cytopathic effect (CPE) was observed (7-10 days post-transfection). Cells were lysed to release intracellular viral particles by three freeze/thaw cycles. The cell lysate was then centrifuged at $3000 \mathrm{rpm}$ for $15 \mathrm{~min}$ at room temperature to pellet the cell debris.

Isolated plaques of the HSV and adenovirus vectors were selected and expanded by infecting the Vero and 293A cells. On the day before infection, $3 \times 10^{6}$ cells per $10-\mathrm{cm}$ plate were 


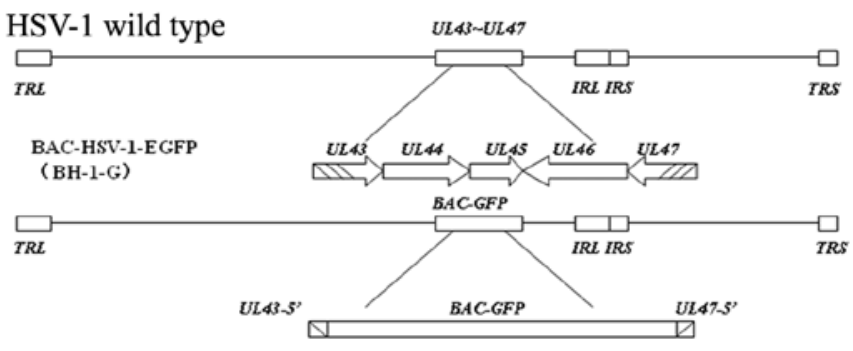

Figure 1. Schematic strategy of the BH-1-G viral vector. BAC-GFP gene was inserted into the genome of HSV-1 F strain to replace several non-essential genes (ul43-ul47) by homologous recombination in Vero cells.

\section{BAC-HSV-1-EGFP(BH-1-G)}

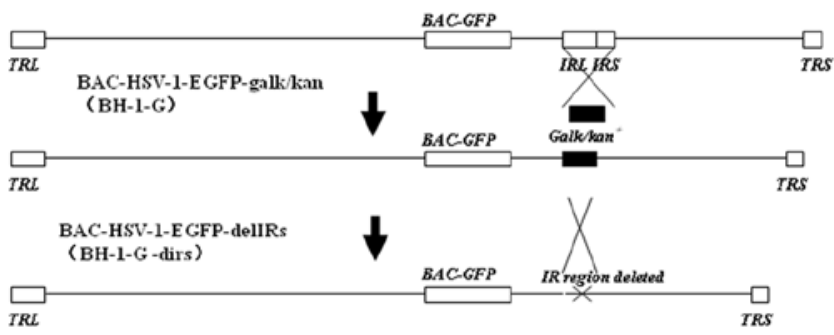

Figure 2. Schematic strategy of BH-1-G-dirs viral vector. BH-1-G-dirs viral vector was constructed by using recombineering technology. IR region was first replaced with Galk/kan ${ }^{+}$by positive selection and then Galk/kan ${ }^{+}$was deleted by negative selection. In this process, UL56 and US1 genes were also deleted partially.

\section{Adv-EGFP(Ad-G)}

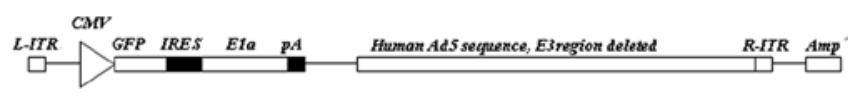

Figure 3. Schematic strategy of Ad-G viral vector. CMV-GFP-IRES-E1apolyA element was cloned to expression vector pDEST by utilizing GATEWAY Cloning System (Gibco BRL). Recombined expression vector was linearized with pacI to transfect into 293A cells to package Adv-GFP virus.

prepared. On the day of infection, the desired amount of virus was added to the cells (MOI of 10) at $80-90 \%$ confluency. The cells and supernatant were harvested, frozen and thawed three times, and the cell lysate was centrifuged. The supernatant containing viral particles was transfered to cryovials in 1-ml aliquots. The viral stocks were stored at $-80^{\circ} \mathrm{C}$, and the titer of the viruses was determined according to TCID50 methods.

Infection with recombinant virus. Lymphoma cells were infected with viral vectors at various MOIs at $37^{\circ} \mathrm{C}$ in a humidified atmosphere of $5 \% \mathrm{CO}_{2}$. Before infection, the lymphoma cells were washed, pelleted at $800 \mathrm{rpm} / 10 \mathrm{~min}$ and re-suspended in PBS. The viruses were diluted in gradient and used to infect cells at the desired MOI. Three hours after infection, the cells were washed with PBS for removal of unattached viruses. Normal medium was added for the further experiments.

Assessment of efficiency of infection. To evaluate the infection efficiency of the HSV-1 vector BH-1-G and adenovirus vector Ad-G in lymphoma cells, the green fluorescent protein (GFP) was used as a reporter, and GFP expression was detected by both flow cytometric analysis and fluorescence microscopy.

FACS analysis. SNK-6, Jurkat, Raji and Hut 78 cells at the log growth phase were centrifuged. The supernatant was discarded, and cells were re-suspend in the desired medium without serum. The cell suspension was seeded in a 24 -well plate at $2 \times 10^{5} /$ well and infected at an MOI of 50 with the viruses (BH-1-G, BH-1-G-dirs and Ad-G), and PBS was used as the control group. The volume of every well was adjusted to $0.9 \mathrm{ml}$. Serum $(100 \mu \mathrm{l})$ was added to all of the experimental wells and the cells were cultured for $4 \mathrm{~h}$ at $37^{\circ} \mathrm{C}$ in a humidified atmosphere with $5 \% \mathrm{CO}_{2}$. The cells were harvested after $24 \mathrm{~h}$, washed twice using cold PBS, centrifuged at $1000 \mathrm{rpm}$ for $5 \mathrm{~min}$ and re-suspend in $500 \mu \mathrm{l} \mathrm{PBS}$, and transferred to a tube used specifically for flow cytometry.

Fluorescence microscopy. An Olympus inverted microscope was used to detect GFP expression. Green fluorescence was readily detectable in the lymphoma cells infected by BH-1-G, BH-1-G-dirs and Ad-G as described above. Twenty-four hours after infection, microphotographs were captured.

Detection of the replication ability. Cells $\left(1 \times 10^{5}\right)$ were seeded on 6-well plates on the day of infection. Viruses (MOI of 1) were used to infect the cells at day 0 time point. Cells and the supernatant were then harvested on day 3 and titrated. The replication ability of viruses was then evaluated according to the titer.

Cytotoxicity assay. The Cell Counting Kit-8 (CCK-8) was used for the cytotoxicity assay of the oncolytic viruses BH-1G-dirs and Ad-G in the lymphoma cells. First, $100 \mu \mathrm{l}$ of cell suspension $\left(1 \times 10^{4}\right.$ cells/well) in a 96 -well plate was needed to be dispensed, and meanwhile viruses were added into the culture media in the plate. The plate was incubated for 4 days in a humidified incubator, and CCK- 8 solution was added to each well for assay. Finally, the absorbance at $450 \mathrm{~nm}$ was measured using a microplate reader after incubating the plate for $4 \mathrm{~h}$.

Statistical analysis. Statistical analysis was performed with one-way ANOVA and the t-test. Statistical significance was set at $\mathrm{P}<0.05$.

\section{Results}

Preparation of virus vectors. Twenty-four hours after infection, the initial CPE was observed in the packaging cells, and complete CPE was observed at $72 \mathrm{~h}$ (Fig. 4). The titer of the harvested viruses was from $5 \times 10^{8}$ to $1 \times 10^{9} \mathrm{PFU} / \mathrm{ml}$. 

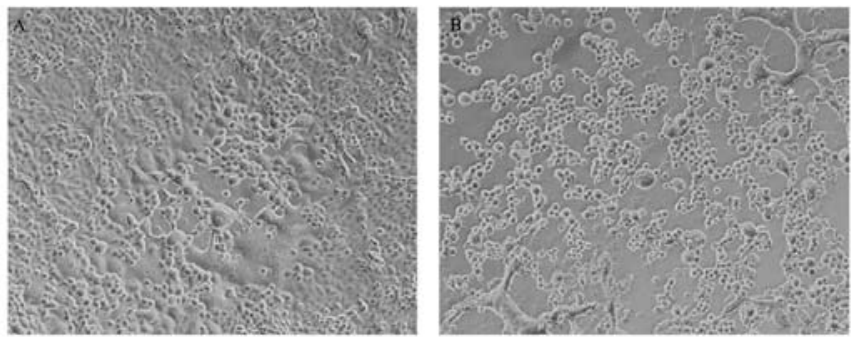

Figure 4. Cytopathic effect (CPE) of the Vero cells after infection. (A) Initial $\mathrm{CPE}$ at $24 \mathrm{~h}$ (B). Complete CPE at $72 \mathrm{~h}$.

Efficiency of infection. Lymphoma cells were infected with the BH-1-G and Ad-G viral vectors derived from HSV-1 and adenovirus, respectively, at a MOI of 50. The observation results showed that the infection statuses of the two viruses in the four lymphoma cell lines were obviously different. Fluorescence intensity of the lymphoma cells after BH-1-G vector infection was higher than that after Ad-G vector infection at the same MOI. Among the four lymphoma cell lines, human cutaneous TL cell line Hut 78 was relatively easier to be infected by the BH-1-G and Ad-G viral vectors, while the human NK/T lymphoma cell line SNK-6 was hardly infected, and the fluorescence intensity did not increase even at a MOI of 1000 (Fig. 5).

To evaluate the efficiency of the viral infection of the lymphoma cells more precisely, $24 \mathrm{~h}$ after infection at a MOI of 50 with the viral vectors, lymphoma cells were harvested and green fluorescence was analyzed by FACS. As shown in Fig. 6, the NK/T lymphoma cell line SNK-6 could not be detected with GFP expression, which indicated that the NK/T lymphoma cell line SNK-6 was not effectively infected with the viral vectors. The results showed that $\sim 57.58$ and $34.67 \%$ of the human cutaneous TL Hut 78 cells infected with the BH-1-G and Ad-G viral vectors, respectively, displayed green fluorescence above the background fluorescence. By comparison, $\sim 14.28$ and $9.28 \%$ of human BL Raji cells infected with the BH-1-G and Ad-G viral vectors expressed green fluorescence, respectively, which was brighter than that detected in the human TL cell line Jurkat with a 10.27 and $5.12 \%$ expression ratio. However, a similar percentage of virus-infected SNK cells displayed an extremely low level of GFP fluorescence of 0.2 and $0.5 \%$ (Fig. 6).

Efficiency of replication. To assess the replication ability of the three types of recombinant viruses (BH-1-G, BH-1-G-dirs and Ad-G) in the lymphoma cells, at days $0,2,4,6,8$ time
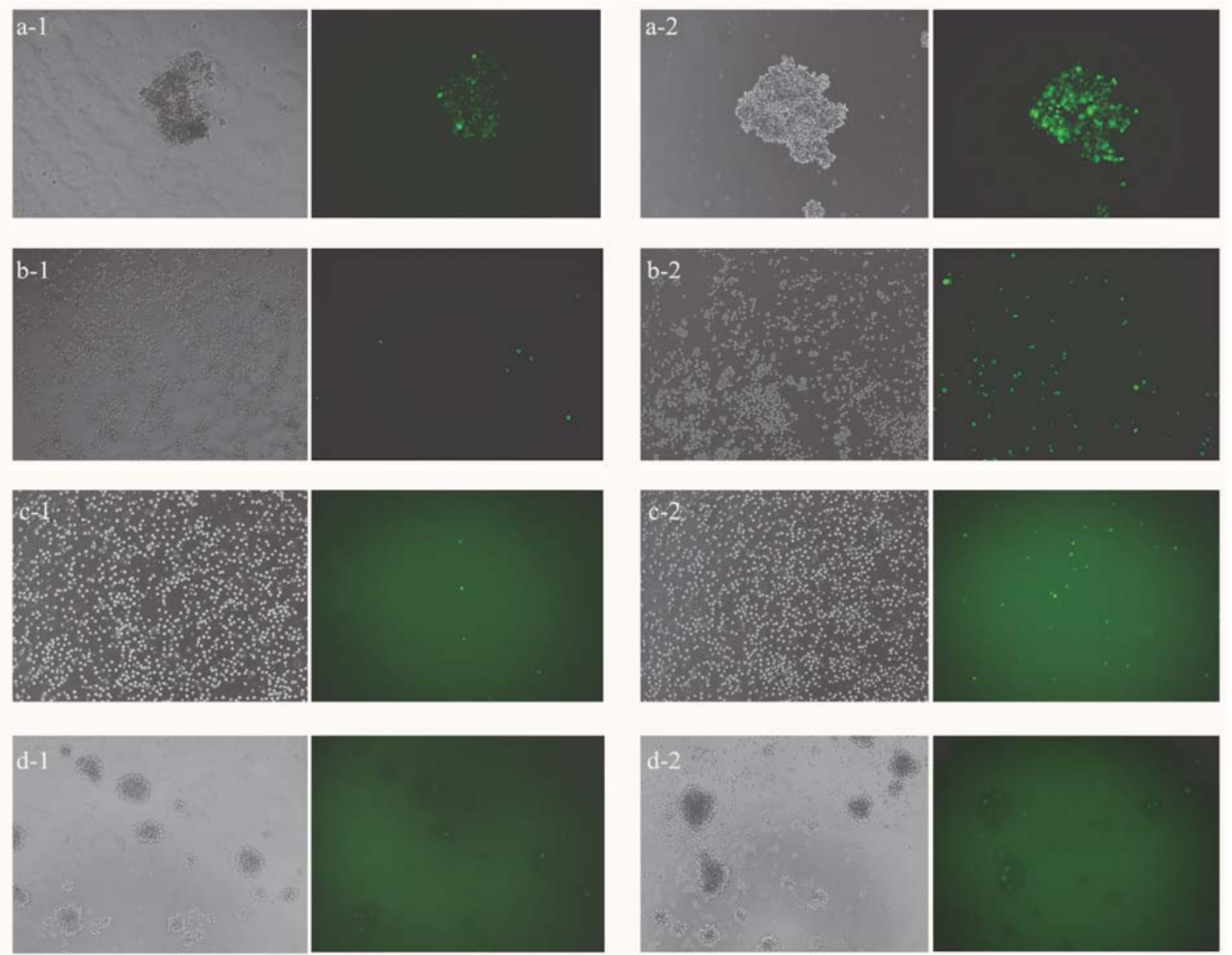

Figure 5. Fluorescence microscopy of GFP expression in infected human lymphoma cells with BH-1-G and Ad-G. Lymphoma cell lines were infected with viral vectors BH-1-G and Ad-G at a MOI of 10 , and $24 \mathrm{~h}$ after infection, microphotographs were recorded. Results showed that the fluorescence intensity of lymphoma cells infected with BH-1-G (a-2, b-2, c-2, d-2) was obviously higher than those infected with Ad-G (a-1, b-1, c-1, d-1). Meanwhile, the fluorescence intensity of Hut 78 (a), Raji (b), Jurkat (c) and SNK-6 (d) cells infected with the same one virus decreased successively. The fluorescence of GFP was barely observed in SNK-6 cells. 

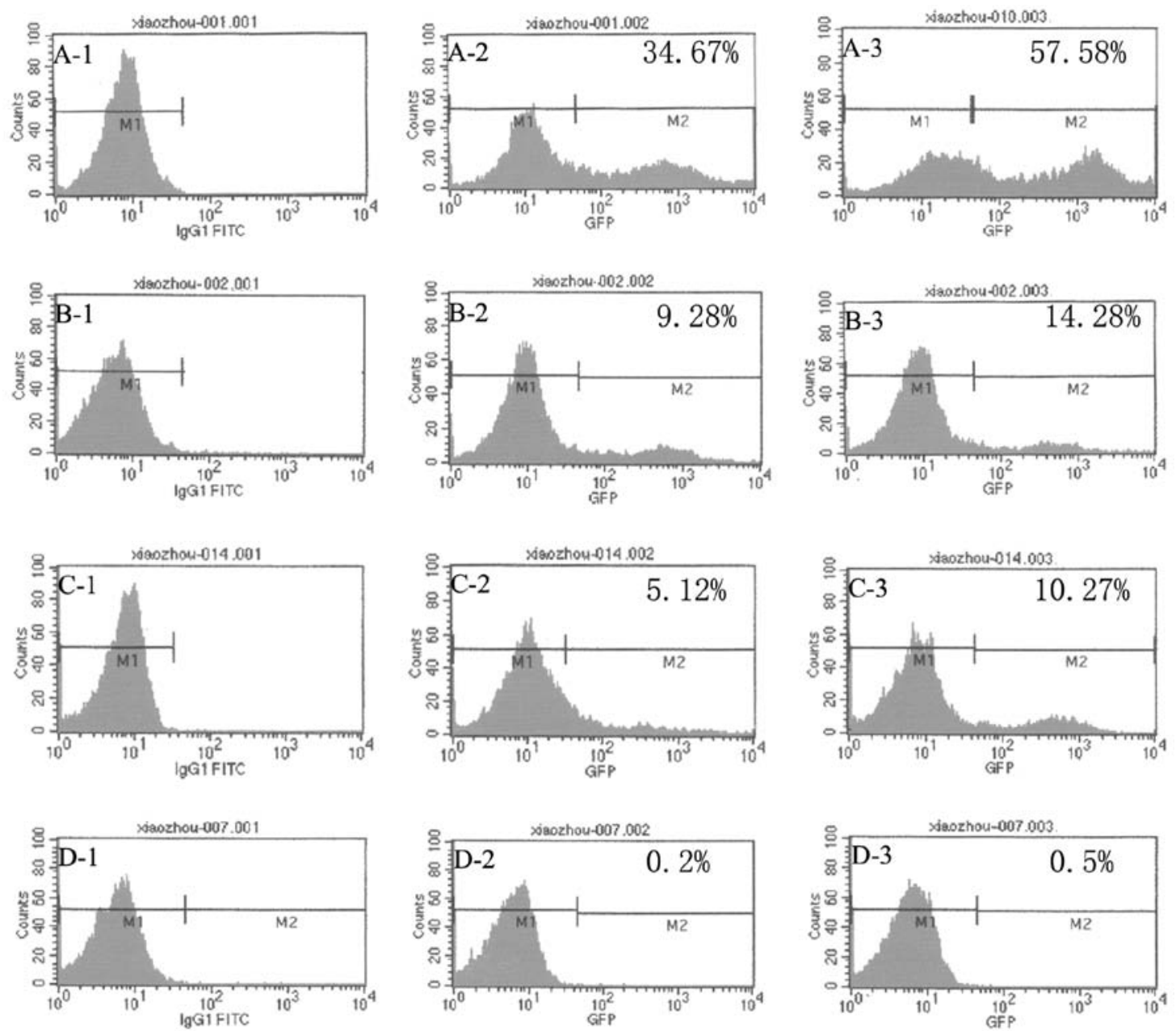

Figure 6. FACS analysis of GFP expression in infected human lymphoma cells. Viruses BH-1-G and Ad-G containing the GFP gene were used to infect human lymphoma Hut 78, Jurkat, Raji and SNK-6 cells. Forty-eight hours after infection at a MOI of 50 with the viral vectors, lymphoma cells were harvested and green fluorescence was analyzed by flow cytometry. The relative numbers of viable cells were then plotted as a function of intensity of green fluorescence. The uninfected cells were also used for comparison (A-1, B-1, C-1 and D-1). Approximately 34.67 and 57.58\% of Hut 78 cells infected with the BH-1-G and Ad-G vectors, respectively, displayed GFP fluorescence over the background (A-2 and A-3). Approximately 14.28 and 9.28\% of the human BL cell line Raji infected with BH-1-G and Ad-G expressed green fluorescence, respectively (B-1 and B-2), which was brighter on average than that detected in the human TL cell line Jurkat with 10.27 and 5.12\% expression ratio (C-2 and C-3). However, a similar percentage of virus-infected SNK cells displayed an extremely low level of GFP fluorescence of 0.2 and $0.5 \%$ (D-2 and D-3).
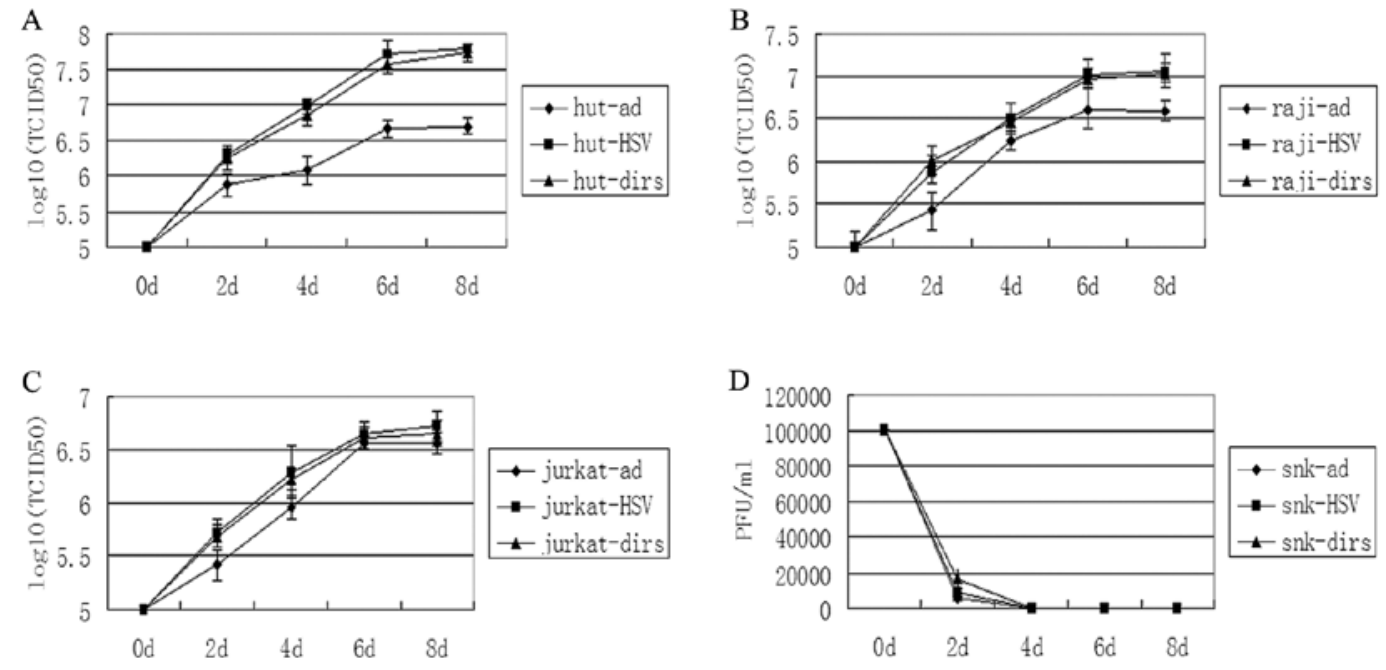

Figure 7. Replication of viral vectors in different lymphoma cell lines. Hut 78, Jurkat, Raji and SNK-6 cells were infected with BH-1-G, BH-1-G-dirs and Ad-G at a MOI of 10. At days 0, 2, 4, 6 and 8, cells and the supernatant were collected and titrated. The result showed that BH-1-G and BH-1-G-dirs replicated effectively in Hut 78, Raji and Jurkat cell lines, and the replication efficiency of HSV-1 vectors was higher than Ad-G (A, B and C). Three types of viruses did not replicate in the SNK cell line, and even were not detected at day 3 after infection (D). 


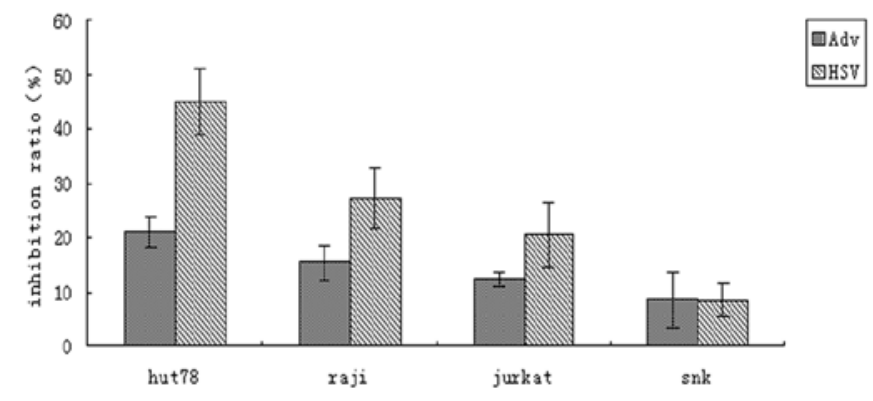

Figure 8. Cytotoxicity of BH-1-G-dirs and Ad-G against three human lymphoma cell lines. Cytotoxicity assay using the CCK-8 Kit was carried out in Hut 78, Jurkat, Raji and SNK-6 cell lines after infection of the cells with BH-1-G-dirs and Ad-G at a MOI of 10. Different responses to BH-1-G-dirs and Ad-G were noted in the different cell lines. Inhibition of $44.9 \%$ of Hut 78 cells, $27.2 \%$ of Raji cells and $20.2 \%$ of Jurkat cells infected with BH-1-G-dirs was noted. By contrast, $21.0 \%$ of Hut 78 cells, $15.3 \%$ of Raji cells and $12.4 \%$ of Jurkat cells infected with Ad-G were inhibited. The difference in inhibition between BH-1-G-dirs and Ad-G was statistically significant $(\mathrm{p}<0.01)$ Although the virus did not replicate in SNK-6 cells, Ad-G and BH-1-G-dirs viruses still exhibited non-specific inhibitory effects (8.5 and 8.2\%).

points after infection at a MOI of 10 , the cells and the supernatant were collected and titrated. The results showed that in human cutaneous TL cell line Hut 78, BH-1-G and BH-1-Gdirs effectively replicated and finally increased by $\sim 500$ times, at least 10-fold more than Ad-G. BH-1-G and BH-1-G-dirs increased by $\sim 100$ and 50 times in thw B cell lymphoma Raji and Jurkat cells, at least 2-fold more than Ad-G which increased 40 and 20 times in the Raji and Jurkat cells. This suggests that the replication ability of $\mathrm{BH}-1-\mathrm{G}$ and $\mathrm{BH}-1-\mathrm{G}-$ dirs was stronger than Ad-G, but the replication ability was similar between BH-1-G and BH-1-G-dirs vectors in the Hut 78, Raji and Jurkat cell lines. (Fig. 7). Three types of viruses did not replicate in the SNK cell line, and even were not detected at day 4 after infection (Fig. 7).

Cytotoxicity assay. Lymphoma cells were infected with BH-1G-dirs and Ad-G at a MOI of 10, and the cytotoxicity was determined using the CCK-8 Kit. Results showed that BH-1G-dirs inhibited the growth of lymphoma Hut 78, Raji and Jurkat cells more effectively than Ad-G (Fig. 8). Human cutaneous TL cell line Hut 78 was the most sensitive to viral oncolysis among the four lymphoma cell lines, and the inhibition ratio of Hut 78 infected by Ad-G and BH-1-G-dirs was 21.0 and $44.9 \%$, respectively. The inhibition ratio of the human BL cell line Raji infected by Ad-G and BH-1-G-dirs was 15.3 and $27.2 \%$, respectively, and the human TL cell line Jurkat was 12.4 and $20.2 \%$, respectively. Although the virus did not replicate in the SNK-6 cells, Ad-G and BH-1-G-dirs still exhibited non-specific inhibitory effects (8.5 and 8.2\%).

\section{Discussion}

Many gene therapy applications require viral vectors, such as adenovirus, herpes simplex viruses, retroviruses and adenoassociated viruses. Adenovirus and HSVs, which can be genetically constructed as oncolytic viral or replicationincompetent vectors, have become the most popular viral vectors.
Lymphoma is a special type of disease comprising a heterogeneous group of lymphoid malignancies. Previous studies have shown that malignant human hematopoietic cells including lymphoma cells are resistant to most viral vectors and only a few reports exist of successful infection into malignant human hematopoietic cells. Diloo et al reported $100 \%$ infection efficiency by replication defective HSV-2 vector into acute myelocytic leukemia (AML), acute lymphocytic leukemia (ALL) and CD34-positive progenitor cells at a MOI lower than 3 (16). Wolfe et al reported that up to $81 \%$ of AML, ALL and CD34-positive stem cells were infected efficiently by HSV-1 vector at a very high concentration of 30 MOI. Higher concentrations of the virus may yield higher infection efficiency (22). To date, there are few research reports on viral vectors that can effectively infect and replicate in lymphoma cells. Herpes simplex viruses (HSVs) may offer numerous advantages as vectors for gene therapy over alternative systems in terms of their titer, the size of the gene insert, safety and a broad tissue tropism, but as yet they have not been proven capable of infection and replication in lymphoma cells.

To examine the infection and replication ability of HSV vectors in the different lymphoma cells, recombinant viruses BAC-HSV-1-eGFP (BH-1-G) and Adv-eGFP virus (Ad-G) inserted with GFP as a reporter, were applied to evaluate the efficiency of viral infection and replication. Microscopy observation methods and flow cytometric assay were used to measure the proportions of cells that expressed GFP for evaluating the infection ability of the viruses targeting the different lymphoma cell lines. This study demonstrated that the efficiency of HSV vector BH-1-G infecting lymphoma cells was higher than adenovirus vector Ad-G (Figs. 5 and 6). As assessed by FACS, 57.58, 14.28 and $10.27 \%$ of Hut 78, Raji and Jurkat cells infected with BH-1-G at an MOI of 50 expressed GFP respectively, while 34.67, 9.28 and 5.12\% cells infected by Ad-G expressed GFP. For the same virus, the infection efficiency also varied between different lymphoma cells, but remained relatively higher for the Hut 78 cells. The efficiency of infection in Hut 78 cells was 10 - to 100 -fold higher than that in the Raji and Jurkat cells at any given MOI. Nevertheless, the SNK-6 cell line was hardly infected by the virus vectors, as the fluorescence of GFP was barely observed in the SNK-6 cells infected with viral vectors at any MOI, and GFP expression was not detected by FACS (Figs. 5 and 6). We also compared the replication efficiency of lymphoma cells with $\mathrm{BH}-1-\mathrm{G}, \mathrm{BH}-1-\mathrm{dirs}$ and Ad-G by using a conventional method TCID 50 for titer measurement. The results showed that the replication ability of $\mathrm{BH}-1-\mathrm{G}$ and $\mathrm{BH}-1-\mathrm{G}-$ dirs was stronger than Ad-G, but the replication ability was similar between the $\mathrm{BH}-1-\mathrm{G}$ and $\mathrm{BH}-1-\mathrm{G}$-dirs vectors in the lymphoma cell lines (Fig. 7). BH-1-G and BH-1-G-dirs replicated effectively and finally increased by $\sim 500$ times, at least 10 -fold more than Ad-G in Hut 78 cells, while BH-1-G and BH-1-Gdirs increased by $\sim 100$ times in the B cell lymphoma cell line Raji and 50 times in Jurkat cells, at least 2-fold more than Ad-G which increased 40 and 20 times in Raji and Jurkat cells. To our surprise, three types of viruses did not replicate in SNK-6 cell line, and also were not detected at day 3 after infection (Fig. 7). We supposed that the viruses may be eliminated by the SNK-6 cells derived from the NK/T cell 
line which limited the replication of the viruses in vitro (23). These unexpected findings suggest that the SNK-6 cell line was unsuitable for use in the viral vector-mediated gene therapy, while the Hut 78 cell line should be an attractive target for viral therapy.

According to the results of the assessment of infection and replication abilities, we demonstrated that the HSV-1 vector infected and replicated in the lymphoma cells more easily than the adenovirus vector. Thus, HSV-1 vectors may be useful in the gene transduction and expression research of lymphoma. A cytotoxicity assay was carried out to evaluate the oncolytic effect of viral BH-1-G-dirs. The results showed that BH-1-Gdirs could kill lymphoma more effectively than the adenovirus vector Ad-G. Hut 78 cells were also more sensitive than the other lymphoma cells to the viral vectors. The inhibition ratio of Hut 78 cells infected with Ad-G and BH-1-G-dirs was 21.0 and $44.9 \%$, respectively. The killing ratio of the human BL cell line Raji infected by Ad-G and BH-1-G-dirs was 15.3 and $27.2 \%$, respectively, and that of human TL cell line Jurkat was 12.4 and $20.2 \%$. Although the virus did not replicate in SNK-6 cells, Ad-G and BH-1-G-dirs viruses still exhibited non-specific inhibitory effects (8.5 and 8.2\%). We supposed that the non-specific inhibition effects were related with the induction of apoptosis after the viral infection $(5,24)$. BH-1-G-dirs was constructed by deleting the internal repeat region including one copy of the RS1, RL1, RL2 gene and mutated UL56, US1 gene for modeling NV1020 oncolytic virus, which has been used in clinical trials and was demonstrated to have significant potential for the treatment of many different types of solid tumors $(20,21)$.

We described in this study three recombinant viruses including BAC-HSV-1-eGFP (BH-1-G), BAC-HSV-1-eGFPdIRs (BH-1-G-dirs) and Adv-eGFP (Ad-G) and demonstrated that the HSV-1-derived vector BH-1-G infected and replicated in human cutaneous TL Hut 78, human BL Raji and human TL Jurkat cell lines more effectively than Ad-G. Compared with Ad-G, the oncolytic ability of BH-1-G was the strongest in the three lymphoma cells noted above. Thus, HSV-1 vectors may become a useful tool for the study of the treatment of lymphoma. Lymphoma is a heterogeneous disease comprising numerous malignancies with different histogenesis and biological characteristics, and different lymphoma cells may have different sensitivities to infection and replication with viral vectors. In our research, the results showed that the difference in sensitivity to virus vectors among the four lymphoma cells was significant. We also found that the human cutaneous TL cell line Hut 78 is a good target for viral therapy due to its high sensitivity to viral vectors, while viral therapy may be not suitable for the human NK/T lymphoma cell line SNK-6 due to its poor sensitivity to viral vectors. Further screening and research in regards to viral therapy for lymphoma both in vitro and in vivo is warranted so that viral therapy can be effectively applied in the treatment of lymphoma.

\section{Acknowledgements}

This study was supported by grants SNK-6 cell line from Norio Shimizu and Yu Zhang of Chiba University. We thank
Dr Zhiqiang Han, Medical College of Zhengzhou University who kindly provided viral vectors.

\section{References}

1. Harris NL, Jaffe ES, Stein H, Banks PM, Chan JK, Cleary ML, Delsol G, De Wolf-Peeters C, Falini B and Gatter KC: A revised European-American classification of lymphoid neoplasms: a proposal from the International Lymphoma Study Group. Blood 84: 1361-1392, 1994.

2. National Cancer Institute sponsored study of classifications of non-Hodgkin's lymphomas: summary and description of a working formulation for clinical usage. The Non-Hodgkin's Lymphoma Pathologic Classification Project. Cancer 49: 2112-2135, 1982.

3. Sacchi S, Federico M, Dastoli G, Fiorani C, Vinci G, Clò V and Casolari B: Treatment of B-cell non-Hodgkin's lymphoma with anti-CD20 monoclonal antibody rituximab. Crit Rev Oncol Hematol 37: 13-25, 2001.

4. Maloney DG, Grillo-López AJ, White CA, Bodkin D, Schilder RJ, Neidhart JA, Janakiraman N, Foon KA, Liles TM, Dallaire BK, Wey K, Royston I, Davis T and Levy R: IDEC-C2B8 (rituximab) anti-CD20 monoclonal antibody therapy in patients with relapsed low-grade non-Hodgkin's lymphoma. Blood 90: 2188-2195, 1997.

5. Peter B, Ingo GH and Schmidt W: Gene therapy of lymphoma. J Hematother Stem Cell Res 11: 457-467, 1997.

6. Peng Z, Yu Q and Bao L: The application of gene therapy in China. IDrugs 11: 346-350, 2008.

7. Grote D, Russell SJ, Cornu TI, Cattaneo R, Vile R, Poland GA and Fielding AK: Live attenuated measles virus induces regression of human lymphoma xenografts in immunodeficient mice. Blood 97: 3746-3754, 2001.

8. Cantwell MJ, Sharma S, Friedmann T and Kipps TJ: Adenovirus vector infection of chronic lymphocytic leukemia B cells. Blood 88: 4676-4683, 1996.

9. Prince HM, Dessureault S, Gallinger S, Krajden M, Sutherland DR, Addison C, Zhang Y, Graham FL and Stewart AK: Efficient adenovirus-mediated gene expression in malignant human plasma cells: relative lymphoid cell resistance. Exp Hematol 26: 27-36, 1998.

10. Huang S, Endo RI and Nemerow GR: Upregulation of integrins alpha $\mathrm{v}$ beta 3 and alpha $\mathrm{v}$ beta 5 on human monocytes and $\mathrm{T}$ lymphocytes facilitates adenovirus-mediated gene delivery. J Virol 69: 2257-2263, 1995.

11. Silver L and Anderson CW: Interaction of human adenovirus serotype 2 with human lymphoid cells. Virology 165: 377-387, 1988.

12. Wang Y, Xue SA, Hallden G, Francis J, Yuan M, Griffin BE and Lemoine NR: Virus-associated RNA I-deleted adenovirus, a potential oncolytic agent targeting EBV-associated tumors. Cancer Res 65: 1523-1531, 2005.

13. Meeker TC, Lay LT, Wroblewski JM, Turturro F, Li Z and Seth P: Adenoviral vectors efficiently target cell lines derived from selected lymphocytic malignancies, including anaplastic large cell lymphoma and Hodgkin's disease. Clin Cancer Res 3: 357-364, 1997.

14. Turturro F, Seth P and Link CJ Jr: In vitro adenoviral vector p53-mediated transduction and killing correlates with expression. Clin Cancer Res 6: 185-192, 2000.

15. Wills KN, Maneval DC, Menzel P, Harris MP, Sutjipto S, Vaillancourt MT, Huang WM, Johnson DE, Anderson SC and Wen SF: Development and characterization of recombinant adenoviruses encoding human p53 for gene therapy of cancer. Hum Gene Ther 5: 1079-1088, 1994.

16. Dilloo D, Rill D, Entwistle C, Boursnell M, Zhong W, Holden W, Holladay M, Inglis S and Brenner M: A novel herpes vector for the high-efficiency transduction of normal and malignant human hematopoietic cells. Blood 89: 119-127, 1997.

17. Wang G, Li G, Liu H, Yang C, Yang X, Jin J, Liu X, Qian Q and Qian W: E1B 55-kDa deleted, Ad5/F35 fiber chimeric adenovirus, a potential oncolytic agent for B-lymphocytic malignancies. Gene Med 11: 477-485, 2009.

18. Peng KW, Ahmann GJ, Pham L, Greipp PR, Cattaneo R and Russell SJ: Systemic therapy of myeloma xenografts by an attenuated measles virus. Blood 98: 2002-2007, 2001.

19. Shen Y and Nemunaitis J: Herpes simplex virus 1 (HSV-1) for cancer treatment. Cancer Gene Ther 13: 975-992, 2006. 
20. Kemeny N, Brown K, Covey A, Kim T, Bhargava A, Brody L, Guilfoyle B, Haag NP, Karrasch M, Glasschroeder B, Knoll A, Getrajdman G, Kowal KJ, Jarnagin WR and Fong Y: Phase I, open-label, dose-escalating study of a genetically engineered herpes simplex virus, NV1020, in subjects with metastatic colorectal carcinoma to the liver. Hum Gene Ther 17: 1214-1224, 2006.

21. Gutermann A, Mayer E, von Dehn-Rothfelser K, Breidenstein C, Weber M, Muench M, Gungor D, Suehnel J, Moebius U and Lechmann M: Efficacy of oncolytic herpesvirus NV1020 can be enhanced by combination with chemotherapeutics in colon carcinoma cells. Hum Gene Ther 17: 1241-1253, 2006.
22. Wolfe D, Wechuck JB, Krisky DM, Goff, JP, Goins WF, Ozuer A, Epperly ME, Greenberger JS, Fink DJ and Glorioso JC: Delivery of herpes simplex virus-based vectors to stem cells. Methods Mol Biol 246: 339-352, 2004.

23. Yasukawa $M$ and Kobayashi Y: Inhibition of herpes simplex virus replication in vitro by human cytotoxic $\mathrm{T}$ cell clones and natural killer cell clones. J Gen Virol 66: 2225-2229, 1985.

24. Han JY, Sloan DD, Aubert M, Miller SA, Dang CH and Jerome KR: Apoptosis and antigen receptor function in $\mathrm{T}$ and $\mathrm{B}$ cells following exposure to herpes simplex virus. Virology 359: 253-263, 2007. 